\title{
Microestrutura, dureza e módulo de elasticidade da liga Ti-12Mo-3Nb na condição tratada
}

\section{Microstructure, hardness and elastic modulus of the treated Ti-12Mo-3Nb alloy}

\author{
José Vicente de Paiva Panaino ${ }^{1}$ \\ Carlos Angelo Nunes ${ }^{2}$ \\ Paulo Roberto $\mathrm{Mei}^{2}$ \\ Luiz Henrique de Almeida ${ }^{3}$ \\ Sinara Borborema Gabriel ${ }^{3}$
}

Artigo

Original

Original

Paper

\section{Palavras-chave:}

Liga de Ti

Microestrutura

Dureza

Módulo de

elasticidade

Biomaterial

\section{Resumo:}

Ligas de titânio do tipo beta têm sido largamente desenvolvidas para aplicações na área biomédica por apresentar uma boa combinação de propriedades, tais como, baixo módulo de elasticidade, alta resistência e boa ductilidade com excelente resistência a corrosão. Devido a isto foi desenvolvida uma nova liga do tipo beta metaestável Ti-12Mo-3Nb. Este trabalho apresenta a microestrutura, dureza e módulo de elasticidade da liga Ti-12Mo-3Nb tratada termicamente em $950 \mathrm{oC}$ por $1 \mathrm{~h}$ sob vácuo. $\mathrm{O}$ material foi caracterizado por difração de raios X, microscopia eletrônica de varredura, medidas de microdureza e de módulo de elasticidade. Os resultados mostraram que a liga Ti-12Mo-3Nb, na condição tratada, pode ser uma promissora alternativa para aplicação biomédica.

\begin{abstract}
In the recent years beta titanium alloys have been developed for biomedical applications due to the combination of its mechanical properties including low elasticity modulus, high strength, and good ductility with excellent corrosion resistance. Due to these applications a new metastable beta titanium Ti-12Mo-3Nb alloy was developed. This work presents the microstructure, hardness and Young's modulus of the Ti-12Mo-3Nb alloy heat treated at $950 \mathrm{oC}$ for $1 \mathrm{~h}$ under high vacuum. The material was characterized by X-ray diffraction, scanning electron microscopy, microhardness measurements and elasticity modulus. The results show that the treated Ti-12Mo-3Nb alloy can be a promising alternative for biomedical application.
\end{abstract}

Key words:

Ti alloy

Microstructure

Hardness

Elastic modulus

Biomaterial

\footnotetext{
Acadêmico do curso de Engenharia de Produção do Centro Universitário de Volta Redonda - UniFOA

${ }^{2}$ Doutor em Engenharia Mecânica pela Universidade Estadual de Campinas - UNICAMP-Campinas

${ }^{3}$ Doutor em Engenharia Metalúrgica e de Materiais pela Universidade Federal do Rio de Janeiro - COPPE/UFRJ - Rio de Janeiro
}

Recebido em 09/2011

Aprovado em 12/2011 


\section{Introdução}

Ligas de Titânio são largamente utilizadas como implantes ortopédicos devido a sua resistência à corrosão, biocompatibilidade, resistência e menor módulo de elasticidade que outros biomateriais metálicos tais como o aço inoxidável e as ligas a base de $\mathrm{Co}-\mathrm{Cr}$ [1]. A liga mais usada em aplicações ortopédicas é a liga Ti-6Al-4V. Embora esta liga apresente um menor módulo de elasticidade comparada com o aço inoxidável e as ligas ade CoCr. Estudos têm mostrado que a liberação de pequenas quantidades de $\mathrm{V}$ no corpo humano pode induzir efeitos citotóxicos [2].

Atualmente, muitas pesquisas estão focando no desenvolvimento de ligas de Ti do tipo beta metaestáveis, compostas de elementos não tóxicos $(\mathrm{Nb}, \mathrm{Mo}, \mathrm{Ta}, . .).[1,3-7]$, pois, as vantagens destas em relação as ligas alfa e alfa + beta (Ti-6Al-4V) inclui seu menor módulo e melhor plasticidade [7-9]. Além disso, as variáveis de processamento podem ser controladas para produzir resultados selecionados [10].

No estudo realizado por Gordin et al [7] no desenvolvmento da liga do tipo beta metaestável Ti-12Mo-5Ta (Ti-6.6Mo-1.47Ta\% at.), aqueles mostraram que esta apresentou um baixo módulo de elasticidade (74 GPa) comparada a liga Ti-6Al-4V. Com o intuito de desenvolver e avaliar a substituição de Ta por $\mathrm{Nb}$ (at.\%), este projeto objetivou o desenvolvimento e caracterização da liga Ti-12Mo$3 \mathrm{Nb}$ (Ti-6.6Mo-1.47Nb\% at.) após tratamento térmico para ser usada como substituto ósseo.

\section{Materiais e métodos}

A liga Ti-12Mo-3Nb foi preparada a partir de $\mathrm{Ti}$, Mo e $\mathrm{Nb}$ de pureza comercial por fusão a arco com eletrodo não consumível de tungstênio em atmosfera de argônio. O lingote obtido foi tratado a $1000^{\circ} \mathrm{C}$ por $24 \mathrm{~h}$ em um forno tubular com resfriamento em água a temperatura ambiente e então forjado a quente (780-860 ${ }^{\circ} \mathrm{C}$ ) até redução em área de $\sim 80 \%$.

A microestrutura da liga foi caracterizada por microscopia eletrônica de varredura. A amostra foi embutida a quente em resina e então polida por técnicas padrões de metalográfia.
As análises de fases da liga nas diferentes condições na forma polida foram realizadas por difração de raios-X (DRX) usando uma Shimadzu modelo DRX 6000 difratometro operada a $40 \mathrm{kV}$ e $30 \mathrm{~mA}$. Um filtro de $\mathrm{CuK}_{\alpha}$ $(\lambda=1.5418 \AA)$ foi usado para este estudo. As fases foram identificadas através da comparação com difratograma simulado. As simulações foram realizadas através do programa Powdercell [11] inserindo dados das fases $\alpha, \beta$ e $\omega$ [12], como grupo espacial, parâmetros de rede e posições atômicas.

A microdureza da liga foi obtida utilizando um equipamento Micromet 2004, Buehler, com uma carga de 100 gf durante $30 \mathrm{~s}$. O valor da microdureza representa a média de 10 medidas. O valor da liga comercial Ti-6Al-4V também foi determinada para comparação.

O valor do módulo de elasticidade da liga foi obtido usando a técnica de indentação instrumentada usando um nanoindentador MTS. $\mathrm{O}$ valor da liga comercial Ti-6Al-4V também foi determinado para comparação. Cada valor do módulo representa a média de 27 medidas.

\section{Resultados e discussão}

A Figura 1 mostra o resultado de difração de raios $\mathrm{X}$ da liga Ti-12Mo-3Nb tratada a $950{ }^{\circ} \mathrm{C} / 1 \mathrm{~h}$ e resfriada em água. Observou-se somente a presença de reflexões referentes a fase $\beta$.

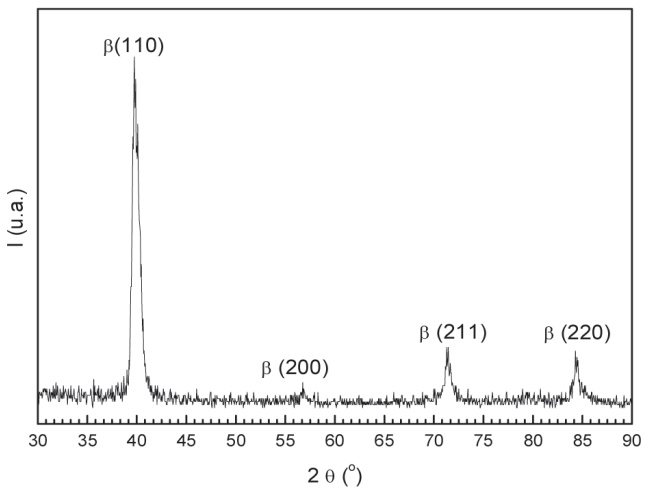

Figura 1- Difratograma de raios $\mathrm{X}$ da liga Ti-12Mo-3Nb tratada a $950^{\circ} \mathrm{C} / 1 \mathrm{~h}$

A Figura 2 mostra a micrografia da liga Ti-12Mo-3Nb na condição tratada. Verificouse a presença de uma monofase que se trata da fase $\beta$ de acordo com os resultados de difração de raios $\mathrm{X}$. 


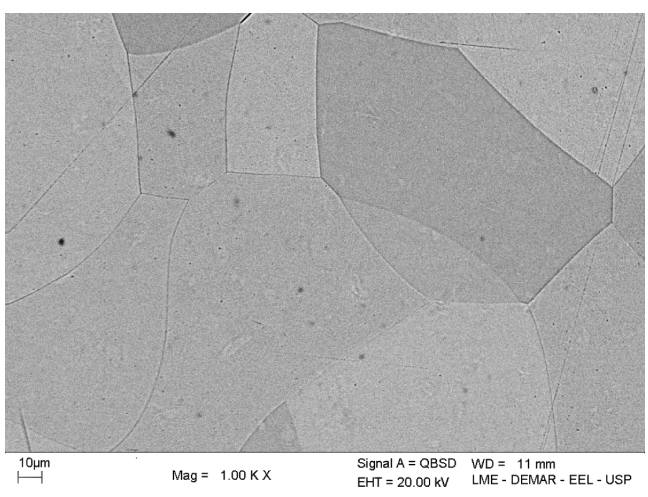

Figure 2- Micrografia da liga Ti-12Mo-3Nb tratada a $950^{\circ} \mathrm{C} / 1 \mathrm{~h}$

A Tabela 1 mostra os valores de módulo de elasticidade, dureza e a razão dureza por módulo de elasticidade das ligas Ti-12Mo-3Nb e Ti-6Al4V . O valor do módulo de elasticidade foi de 105 GPa para a liga $\beta$-Ti-12Mo-3 $\mathrm{Nb}$ enquanto foi de $140 \mathrm{GPa}$ para a liga Ti-6Al-4V. Este valor foi aproximadamente $25 \%$ menor que o obtido para a liga Ti-6Al-4V, enquanto que para a liga Ti12Mo-5Ta, em comparação com a liga Ti-6Al$4 \mathrm{~V}$, este valor foi aproximadamente $38 \%$ menor [7]. Já os valores de dureza da liga Ti-6Al-4V foi de $337 \mathrm{HV}$, enquanto para a liga Ti-12Mo$3 \mathrm{Nb}$ foi $371 \mathrm{HV}$. Já a razão dureza por módulo de elasticidade (medida de desempenho das ligas [4]), a liga Ti-12Mo-3Nb apresentou uma razão dureza por módulo de elasticidade superior a liga comercialmente usada (Ti-6Al-4V).

Tabela 1- Valores de dureza, modulo de elasticidade e a razão dureza/ módulo de elasticidade das ligas estudadas.

\begin{tabular}{cccc}
\hline Ligas & Microdureza (HV) & Módulo de elasticidade (GPa) & Razão dureza/módulo \\
\hline Ti-12Mo-3Nb & 371 & 105 & 3,53 \\
Ti-6Al-4V & 337 & 140 & 2,41 \\
\hline
\end{tabular}

\section{Conclusão}

A liga Ti-12Mo-3Nb mostrou uma microestrutura monofásica beta. O valor do módulo de elasticidade da liga foi aproximadamente 25 $\%$ menor que o obtido para a liga Ti-6Al-4V.

Em relação à substituição de Ta por $\mathrm{Nb}$ (\% at.), na liga a base de Ti-12Mo, verificouse, de acordo com as técnicas de caracterização utilizadas, que as duas ligas apresentaram uma microestrutura monofásica beta, porém, o módulo de elasticidade da liga Ti-12Mo-5Ta foi menor que o obtido para a liga desenvolvida neste trabalho.

Os resultados indicam que a liga Ti-12Mo $3 \mathrm{Nb}$ pode ser uma promissora alternativa para aplicação biomédica quando comparada a liga Ti-6Al-4V, pois esta apresentou uma razão dureza/módulo de elasticidade superior a esta liga.

Acknowledgements: Agradecemos ao Prof. Sérgio Camargo do Laboratório de superfícies do Programa de Engenharia Metalúrgica e de Materiais da COPPE/UFRJ. 


\section{References}

1. [1] Y. L. Zhou, M. Niinomi, T. Akahori, , H. Fukui , H. Toda, Mater Sci Eng A. $398,(2005)$ 28-36.

2. [2] D. Raabe, B. Sander, M. Fria'k, D. Ma, J. Neugebauer, Acta Mater. 55, (2007) 4475-4487.

3. [3] S. B. Gabriel, C. A. Nunes, G. A. Soares, Artif Organs. 32 (2008) 299-304.

4. [4] S. B. Gabriel, J. Dille, C. A. Nunes, G. A. Soares, Mat. Research. 13 (2010) 1-5.

5. [5] A. Cremasco, W. H. Osório, C. M. A. Freire, A. Garcia, R. Caram, Electrochim Acta. 53 (2008) 4867-4874.

6. [6] C.R.M. Afonso, G.T. Aleixo, A.J. Ramirez, R. Caram, Mater Sci Eng C. 27, (2007) 908-913.
7. [7] D.M. Gordin, T. Gloriant, Gh. Nemtoi, R. Chelariu, N. Aelenei, A. Guillou, D. Ansel, Materials Letters, 59, (2005) 2959-2964,

8. [8] R. Banerjee, S. Nag, H. L. Fraser, Mater Sci Eng C. 25 (2005) 282-289.

9. [9] W. Xu, K. B. Kim, J. Das et al., Scr. Mater. 54 (2006) 1943-1948.

10. [10] N. T.C. Oliveira, G. Aleixo, R. Caram, A. C. Guastaldi, Mater Sci Eng A. 452-453, (2007) 727-731.

11. [11] W. Kraus and G. J.Nolze, Powdercell - a program for the representation and manipulation of crystal structure and calculation of the resulting X-ray powder patterns. J Appl Crystallogr. 29 (1996) 301-303.

12. [12] P. Villars and L. D. Calvert, Pearson's handbook of crystallographic data for intermetallic phases. second ed., Metals Park Ohio, 1991.

\section{Endereço para Correspondência:}

Sinara Borborema Gabriel

sinarab@msn.com

Centro Universitário de Volta Redonda - UniFOA

Campus Universitário Olezio Galotti

Av. Paulo Erley Abrantes, 1325

Três Poços - Volta Redonda - RJ

CEP: $27.240-560$

Informações bibliográficas:

Conforme a NBR 6023:2002 da Associação Brasileira de Normas Técnicas (ABNT), este texto científico publicado em periódico eletrônico deve ser citado da seguinte forma: PANAINO, José Vicente de Paiva; NUNES, Carlos Angelo; MEI, Paulo Roberto; ALMEIDA, Luiz Henrique de; GABRIEL, Sinara Borborema. Microestrutura, dureza e módulo de elasticidade da liga Ti-12Mo-3Nb na condição tratada. Cadernos UniFOA. Volta Redonda, Ano VI, n. 17, dezembro 2011. Disponível em: <http://www.unifoa.edu.br/cadernos/ 